\title{
The dependence of the IMF on the density- temperature relation of pre-stellar gas
}

\author{
S. Kitsionas ${ }^{1}$, A. P. Whitworth ${ }^{2}$, R. S. Klessen ${ }^{3}$ and A.-K. Jappsen ${ }^{1}$ \\ ${ }^{1}$ Astrophysikalisches Institut Potsdam, An der Sternwarte 16, D-14482 Potsdam, Germany \\ email: skitsionas@aip.de \\ ${ }^{2}$ School of Physics and Astronomy, Cardiff University, P.O. Box 913, CF24 3YB Cardiff, U.K. \\ ${ }^{3}$ Institut fuer Theoretische Astrophysik, Universitaet Heidelberg, Albert-Ueberle-Strasse 2, \\ D-69120 Heidelberg, Germany
}

\begin{abstract}
It has been recently shown by several authors that fragmentation of pre-stellar gas (i.e. at densities from $10^{4}$ to $10^{10}$ particles $\mathrm{cm}^{-3}$ and temperatures of order 10-30 K) depends on the gas thermodynamics much more than it was anticipated in earlier studies, in which only an isothermal behaviour has been assumed for the gas. Here we review the results of a number of numerical hydrodynamic simulations (e.g. Li et al. 2003, Jappsen et al. 2005, Bonnell et al. 2006) in which departure from isothermality has been attempted by employing a polytropic equation of state (eos) with exponent different from unity. In particular, in these studies it has been shown that the dominant fragmentation scale of pre-stellar gas, and hence the peak of the initial mass function (IMF), depends on a polytropic exponent that changes value, from below to above unity, at a critical density (Larson 2005). Furthermore, this piecewise polytropic eos depends on the gas metallicity and fundamental constants. Therefore, the peak of the IMF depends, in turn, also on the gas metallicity and fundamental constants rather than on initial conditions, as it has been previously suggested (e.g. Larson 1995). Hence, we are for the first time in a position to infer theoretically the notion of a universal IMF (at least for its low-mass end).
\end{abstract}

We also present two test cases in which a non-isothermal eos has been used in the context of smoothed particle hydrodynamic ( $\mathrm{SPH}$ ) numerical simulations. In the first case star formation is triggered by means of low-mass clump collisions. These calculations have shown that clump collisions can be a relatively efficient mechanism for the formation of solar-mass protostars and their lower-mass companions (efficiency greater or of order 20-25\%; Kitsionas \& Whitworth 2006). We have also found that in such collisions protostars form mainly by fragmentation of dense filaments along which it is likely that pairs of protostars capture each other in close binaries surrounded by circumbinary discs. In the second case, the use of a polytropic eos with a varying exponent appropriate for the metallicity of starburst regions (Spaans \& Silk 2000, 2005) is shown to be sufficient to obtain a top heavy IMF similar to that observed e.g. in the Galactic centre (Klessen, Spaans \& Jappsen 2006). These are preliminary results in the direction of revisiting earlier isothermal calculations that were resolving all densities up to the opacity limit for fragmentation (e.g. Bate et al. 2002ab, 2003), this time also taking into account the thermal properties of the gas in the density range between $10^{4}$ and $10^{10}$ particles $\mathrm{cm}^{-3}$. The next step would be to include self-consistent radiation transport in the calculations, the first attempts for which are already in the making (e.g. Whitehouse \& Bate 2004).

Keywords. equation of state, hydrodynamics, method: numerical, stars: formation, stars: mass function 